\title{
Nitrogen Laser Emissions of Short and Long Durations Generated in Air
}

\author{
Mladen M. Kekez ${ }^{(1)}$ and David M. Villeneuve
}

\begin{abstract}
This article describes two sets of experiments. An eight-stage, pulse-forming network (PFN) Marx generator of 50- $\Omega$ internal impedance produces a "square"-shaped voltage waveform and induces multiphoton ionization, excitation of molecules and ions to generate laser pulses of long $(<3 \mu \mathrm{s})$ and short ( $\sim 10 \mathrm{~ns})$ durations in air, when the principal laser line of the $\mathrm{N}_{2}$ laser at $337.1 \mathrm{~nm}$ was observed. The visible blue laser lines coming from the first negative band of $\mathrm{N}_{2}^{+}$ions due to the $\mathrm{B}^{2} \sum_{u}^{+} \rightarrow \mathrm{X}^{2} \sum_{g}^{+}$transition at 391.4 and $427.8 \mathrm{~nm}$ were studied. The wideband (300-800 $\mathrm{nm}$ ) Schott BG 39 interference filter was also employed to examine whether some other lines participate in the emission being generated. The scaling law was derived describing the attenuation of the emission versus the distance for long-duration pulses $(<3 \mu \mathrm{s})$ at $337.1 \mathrm{~nm}$. The second set of experiments was introduced to support the data obtained. It offers information pertinent to the visible blue line emissions as well as to the photoionization processes, lighting phenomena, and processes occurring at high altitudes.
\end{abstract}

Index Terms-Corona, lighting, nitrogen laser, photoionization, spark channels.

\section{INTRODUCTION}

$\mathbf{N}$ LASER is a useful source of pulsed ultraviolet (UV) laser radiation. With the low $(<1 \Omega)$-internal impedance Blumlein driver, the commercial $\mathrm{N}_{2}$ laser operates usually with voltages ranging from 8 to $14 \mathrm{kV}$ and pressures between 70 and 250 torr of $\mathrm{N}_{2}$. Under optimum conditions, the laser provides pulses with energies of $<30 \mathrm{~mJ}$ and the width of the pulse not exceeding 19 ns [1].

The classical $\mathrm{N}_{2}$ laser is pumped by an electrical discharge to create numerous filamentary streamer/corona channels in the glow-like discharge formed between the two parallel electrodes. The gas discharges formed in $\mathrm{N}_{2}$ and/or air provides the $\mathrm{N}_{2}$ laser gain medium. The preionization had to be applied to produce a semi-uniform glow-like column [1]-[4].

The duration of the laser pulse as a function of pressure is proportional to the time interval of the voltage fall from the full value of the voltage supplied by the Blumlein driver to almost zero value, due to the formation of the spark channel. When the $\mathrm{N}_{2}$ laser is made to operate at a high ( $<760$ torr)

Manuscript received September 14, 2019; revised December 9, 2019; accepted January 20, 2020. Date of publication February 19, 2020; date of current version March 10, 2020. The review of this article was arranged by Senior Editor S. J. Gitomer. (Corresponding author: Mladen M. Kekez.)

Mladen M. Kekez, deceased, was with High-Energy Frequency Tesla Inc., Ottawa, ON K1H 7L8, Canada (e-mail: daniel.kekez@utoronto.ca).

David M. Villeneuve is with the National Research Council of Canada, Ottawa, ON K1A 0R6, Canada, and also with the Department of Physics, Faculty of Science, University of Ottawa, Ottawa, K1N 6N5, Canada (e-mail: david.villeneuve@nrc-cnrc.gc.ca).

Color versions of one or more of the figures in this article are available online at http://ieeexplore.ieee.org.

Digital Object Identifier 10.1109/TPS.2020.2969420 pressure, the excitation of the $\mathrm{N}_{2}$ molecules, and consequently the laser emission would yield only short duration light pulses, from one nanosecond down to some tens of picoseconds.

From the research and development on $\mathrm{CO}_{2}$ lasers, it was learned that the delay in the transition from the glow-like discharge to a fully developed spark channel is possible, when the characteristic impedance of the driver is of relatively large value $(>10 \Omega)$.

To explore the importance, the nine-stage Marx generator of 18- $\Omega$ internal impedance was used in [5]. The electromagnetic radiation pulses were observed both at the single microwave frequency close to $1 \mathrm{GHz}$ and the pulses of long duration $(<220 \mathrm{~ns})$ at $337.1 \mathrm{~nm}\left(=\right.$ prominent $\mathrm{N}_{2}$ laser line) in the pressure range from 100 to 200 torr in nitrogen, and also in air, without the use of the laser resonator and mirrors. These radiation pulses can be considered as the coherent radiation due to the maser action and the laser action, respectively [5].

To explore further the importance of impedance, an eightstage, pulse-forming network (PFN) Marx generator with $50-\Omega$ internal impedance was used in [6]. The pulses of nitrogen emission at $337.1 \mathrm{~nm}$ of long duration $(<3 \mu \mathrm{s})$ and of short duration $(\sim 10 \mathrm{~ns})$ were obtained.

This article is organized as follows. For both the shortand long-duration pulses, attention is given to the electronicvibrational-rotational transitions $\left(\mathrm{C}^{3} \Pi_{u} \rightarrow \mathrm{B}^{3} \Pi_{g}\right)$ of the second positive system of a nitrogen molecule with a characteristic peak at $337.1 \mathrm{~nm}$. The visible blue laser lines coming from the first negative band of $\mathrm{N}_{2}^{+}$ions due to the $\left(\mathrm{B}^{2} \sum_{u}^{+} \rightarrow\right.$ $\mathrm{X}^{2} \sum_{g}^{+}$) transition at $391.4 \mathrm{~nm}$ and $427.8 \mathrm{~nm}$ were studied. Emphasis is given toward understanding the attenuation of the $337.1 \mathrm{~nm}$ line (= principal laser line of $\mathrm{N}_{2}$ laser) of longduration pulses $(<3 \mu \mathrm{s})$ as it propagates in air

To support the data obtained, the second set of experiments was introduced to provide additional information regarding the visible blue line emissions, as well as regarding the processes of photoionization, ionization by the electric field, lighting phenomena, and processes occurring at high altitudes.

\section{EXPERIMENTAL SETUP}

In this article, an eight-stage PFN Marx generator described in [6] was used.

When the nipple 4 in the experimental setup (see Fig. 1) is removed and the voltage impulse of negative polarity from an eight-stage PFN Marx generator is applied, it creates the spark channel breakdown between the copper wire 6 and the metallic electrode 5. The gap between the tip of the wire 6 and 


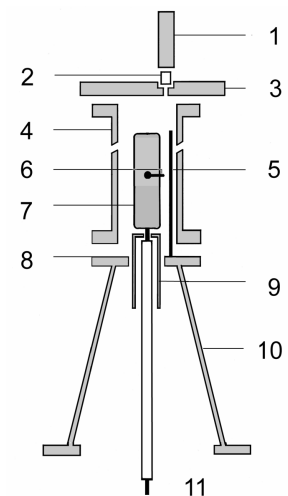

Fig. 1. Schematics of the experimental setup. 1: Hamamatsu R 727 photodiode, 2: optical filter, 3: Plexiglas flange with an aperture (i.e., orifice) of $6.25 \mathrm{~mm}$ and the additional recess to accommodate the filter, $4: 23 / 4$-in (Varian) nipple, 5: metallic post, 6: copper wire attached to the central electrode, 7: central electrode, 8: upper edge of the conical structure, 9: Plexiglas thimble with a hole in the center machined to fit well the polyethylene insulation of the coaxial cable, 10: conical structure connected to the ground of the Marx generator, and 11: inner conductor of the coaxial cable with connection to the generator.

the metallic electrode 5 is $\sim 3 \mathrm{~mm}$. The separation between the metallic electrode 5 and the metallic post 7 is varied between 4 and $7 \mathrm{~mm}$ until the reproducible data for the emission at $337.1 \mathrm{~nm}$ of short duration is obtained. The diameter of the central electrode 7 is $15.9 \mathrm{~mm}$ with the height set initially at $55 \mathrm{~mm}$ and later made to be $80 \mathrm{~mm}$ in length. The metallic post is of $2.5-\mathrm{mm}$ diameter.

The thimble 9 machined out of Plexiglas was placed over the polyethylene surface of the RG $217 / \mathrm{U}$ coaxial cable to minimize the harmful effect of corona tracking along the surface of the cable. The height of the thimble above the upper edge of the conical structure 8 was $32 \mathrm{~mm}$.

The precise alignment in the vertical direction is required, so that the photodiode 1 indicated in Fig. 1 can view the glowlike discharges formed between the central electrode 7 and the metallic post 5 and/or the nipple 4 when it is used.

\section{Diagnostics}

The diagnostic tools are a Hamamatsu R 727 photodiode for recording $\mathrm{N}_{2}$ emissions, the Rogowski coil, and a 3-GHz Tektronix oscilloscope: Model: TDS 694C to record the data. The Rogowski coil (= wide band current transformer), Model 110 made by Pearson Electronics Inc., Palo Alto, USA, was inserted in the space between the points: 10 and 11, as indicated in Fig. 1.

The Hamamatsu R 727 photodiode (with a rise time of $<1 \mathrm{~ns}$ ) was placed on top of the optical interference filter, and both were located on top of the Plexiglas flange 3 shown in Fig. 1.

The optical $337.1 \pm 2 \mathrm{~nm}$ filter of 11.8 -mm diameter was made by Edmund Optics, USA. Other bandpass filters, all with a clear aperture diameter of $21 \mathrm{~mm}$, at $390 \pm 2,420 \pm 2$, and $430 \pm 2 \mathrm{~nm}$ were made by Thorlabs, USA. All these narrow bandpass filters have the full width at half maximum (FWHM) of $10 \mathrm{~nm}$. The wideband (300-800 nm) Schott BG 39 interference filter of $5.08 \mathrm{~mm}$ diameter was made by Lattice Electro Optics, USA.
To avoid the interference problems mentioned in [5], the laser signals recorded by the photodiode have been viewed with the aid of a low (150 MHz)-pass filter.

\section{EXPERIMENTAL RESULTS}

The emissions recorded with four narrow bandpass interference filters and a wideband BG 39 filter are shown in Figs. 2-4. The analysis of the data obtained is given in Figs. 5 and 6.

It may be useful to understand the mechanism of the laser beam generation of short duration, as registered with the aid of a 337.1-nm filter. When the Plexiglas tube was replaced with the nipple 4 shown in Fig. 1, the data were obtained and are shown in Fig. 2. When an eight-stage PFN Marx generator is energized, the spark breakdown takes place between the metallic post 5 and the copper wire attached to the central electrode 6 . The spontaneous emission coming from the spark channel causes the photoionization of air.

In general, photoionization from excited states is the mechanism of gas breakdown by the visible and UV spectrum electromagnetic waves of high intensity, without the benefit of ionization by the external electric field [7]. As a function of gas density/pressure, the electron densities produced by the UV source have been derived for several gases and gas mixtures using, for example, the microwave interferometry method [8]. The microsecond time scale was applied in these measurements.

The detailed studies of plasma density created by photoionization versus distance from the spark channel $d$ reported in [8] and [9] show that the experimental data can be summarized by the following expression:

$$
\text { Plasma density }\left(\mathrm{cm}^{-3}\right)=[A \text { exp. }(-d / k)] / d^{2} .
$$

At $6 \mathrm{~cm}$ away from the spark channel, the plasma density reaches the level of $10^{11} \mathrm{~cm}^{-3}$, and at $30 \mathrm{~cm}$ away, the density falls to $2 \times 10^{8} \mathrm{~cm}^{-3}$. These data were obtained for the typical $\mathrm{CO}_{2}$-laser mixture, where a small $(\sim 10 \mathrm{ppm})$ amount of organic additive (= tripropylamine) was added. In [9], the additive was absent and still (1) was confirmed to be valid. Expression (1) was derived for two sets of the $\mathrm{CO}_{2}$-laser mixture. In one set, the constant $k$ has the value of $4.9 \mathrm{~cm}$, and in the other set, $k$ is $5.5 \mathrm{~cm}$.

The conclusion of these studies shows that in a multiple-step process, the effective photoionization cross section is reduced, and this would allow deep penetration of the incident photons and production of photoelectrons over a large volume.

The resistance of the filamentary spark channel formed initially between the point 6 and the metallic post 5 is initially large, so that part of the voltage pulse from the generator will traverse between the metallic post 5 and the central electrode 7 until it reaches the end of this electrode. The wave will see the open circuit and will reflect back toward the coaxial structure 10 and the inner part of the coaxial cable 11. There, we do not have the matching impedance condition and the wave will travel forth and back, creating the electron-impact ionization in the space between the metallic post 5 and the central electrode 7 , thereby enhancing the electron density that is initially generated by the photoionization process. 

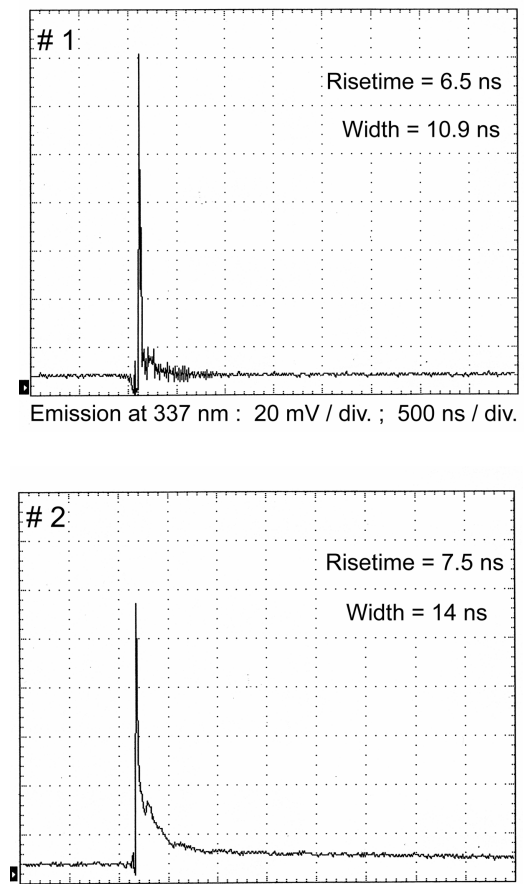

Emission at $390 \mathrm{~nm}$ : $20 \mathrm{mV} /$ div. ; $500 \mathrm{~ns} /$ div.
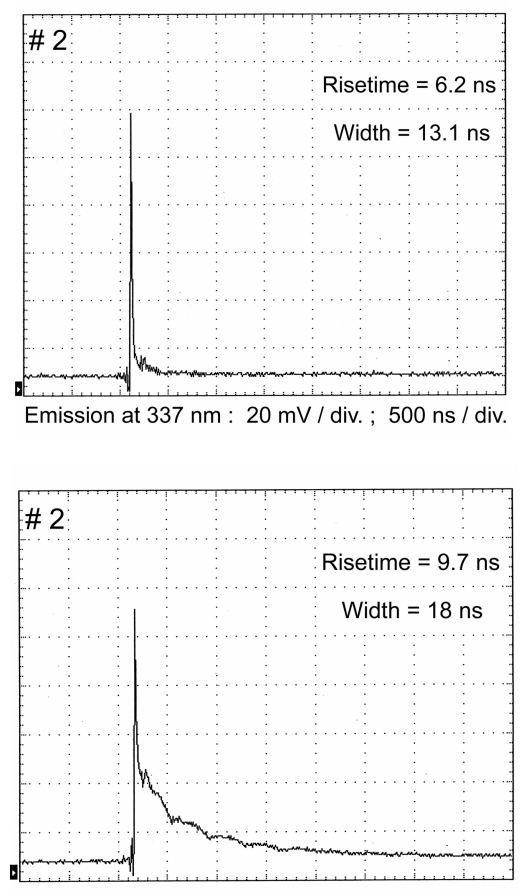

Emission at $420 \mathrm{~nm}$ : $20 \mathrm{mV} /$ div. ; $500 \mathrm{~ns} /$ div.
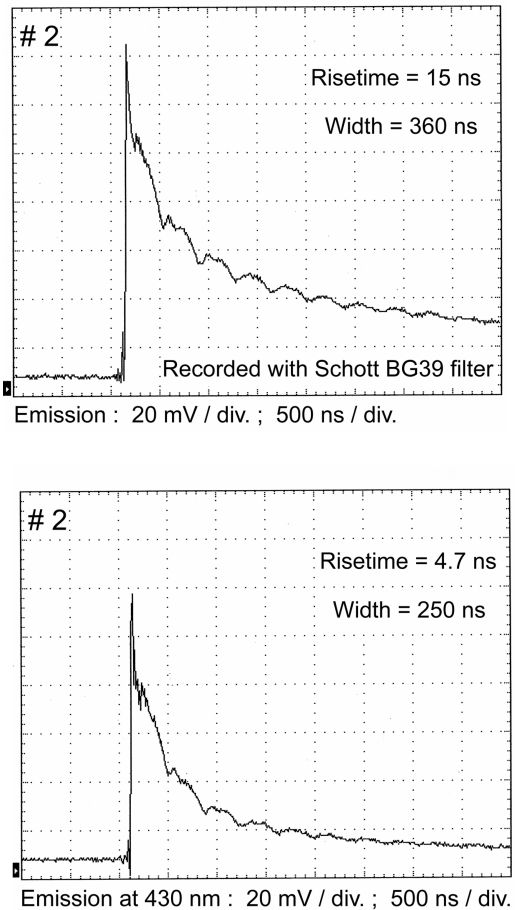

Fig. 2. Experimental setup to generate the emission at $337.1 \mathrm{~nm}$ of short duration. Arrangement \#1 is for the setup when the photodiode is placed at the exit of the setup. Number \#2 is for the arrangement when the photodiode is placed $14 \mathrm{~cm}$ away. The peak amplitude of the emission measured with the aid of a Schott BG39 wideband filter is $143.2 \mathrm{mV}$ in arrangement \#2. The average peak amplitude of emission (= the sum of peak amplitudes of the emission recorded with the aid of narrowband filters at 337, 390, 420, and $430 \mathrm{~nm}$ and divided by 4) is $109.5 \mathrm{mV}$ in arrangement \#2. The ratio between these two quantities is $143.3 / 109.5=1.3078$.

The electric field of the voltage pulse could be close to the voltage breakdown potential, so the filamentary corona-like channel may also be formed at the far end of the central electrode 7 toward the metallic post 5. The UV preionization provides and maintains the necessary ionization in the gas to carry the current and provides electrical excitation of the molecules. The plasma produced by the UV preionization was also augmented by the applied electric fields via the Townsend multiplication parameters (defined via the parameter related to the ratio between the electric field and the neutral gas density) together with the parameters related to the high-speed (runaway) electron production.

In time, the equilibration between the electrons and ions in the spark channel will be established and the resistance of the spark channel will fall to a smaller value, hence the heating of the electron gas will cease, and consequently the electrical excitation of the molecules will also cease. When the low $(<1 \Omega)$-internal impedance Blumlein driver is used, the current flowing in the spark channel is of large amplitude and the equilibration between the electrons and ions in the spark channel will take place in much shorter period of time. This offers an explanation why the nitrogen laser emission with the Blumlein driver is of short duration, when the system is made to operate at a high $(<760$ torr $)$ pressure.

As stated before, the discharge formed between the central electrode 7 and the metallic post 5 has the feature of the glow-like column. The initial filamentary channel performs the following two functions: 1) to provide the preionization in post 5 in order to have a glow-like column and 2) to provide an incident signal of sufficient strength/number of photons needed for the onset of the stimulated (laser) emission. These findings are in accord with the observation stated in [3], i.e., the details of the geometrical arrangement of the electrodes govern whether the stable emission will occur in their classical Blumlein $\mathrm{N}_{2}$ laser. In some of their arrangements, there was no emission, although the glow-like discharge was present between the electrodes.

By carefully adjusting the separation between the metallic post 5 and the central electrode 7 , the narrow pulse of $\sim 10$-ns duration at $337.1 \mathrm{~nm}$ and of high intensity was generated. As a function of distance, it was observed that the pulses are capable of propagating well over 1-m distance. However, the amplitude of the pulse falls, as the pulse was observed further away from the exit.

To have reproducible pulses, it is advisable to tune the system to the moderate level of radiation output at $337.1 \mathrm{~nm}$, as shown in Fig. 2. Setting the system to generate the largest possible amplitudes, the system would give considerable variation in the radiation output shot to shot.

The electronic-vibrational-rotational transitions $\left(\mathrm{C}^{3} \Pi_{u} \rightarrow\right.$ $\mathrm{B}^{3} \Pi_{g}$ ) of the second positive system of a nitrogen molecule with a characteristic peak at $337.1 \mathrm{~nm}$ were of primary interest in this article. The visible blue laser lines from the first negative band of $\mathrm{N}_{2}^{+}$ions due to the $\left(\mathrm{B}^{2} \sum_{u}^{+} \rightarrow \mathrm{X}^{2} \sum_{g}^{+}\right)$ transition at $391.4 \mathrm{~nm}$ and $427.8 \mathrm{~nm}$ were also considered. Because the line at $427.8 \mathrm{~nm}$ has a broad width, it was decided to view this line with the aid of two filters at 420 and $430 \mathrm{~nm}$. 


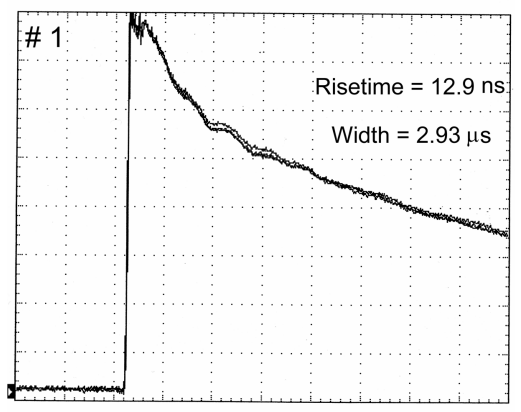

Emission at $337 \mathrm{~nm}$ : $20 \mathrm{mV} /$ div. ; $500 \mathrm{~ns} /$ div.
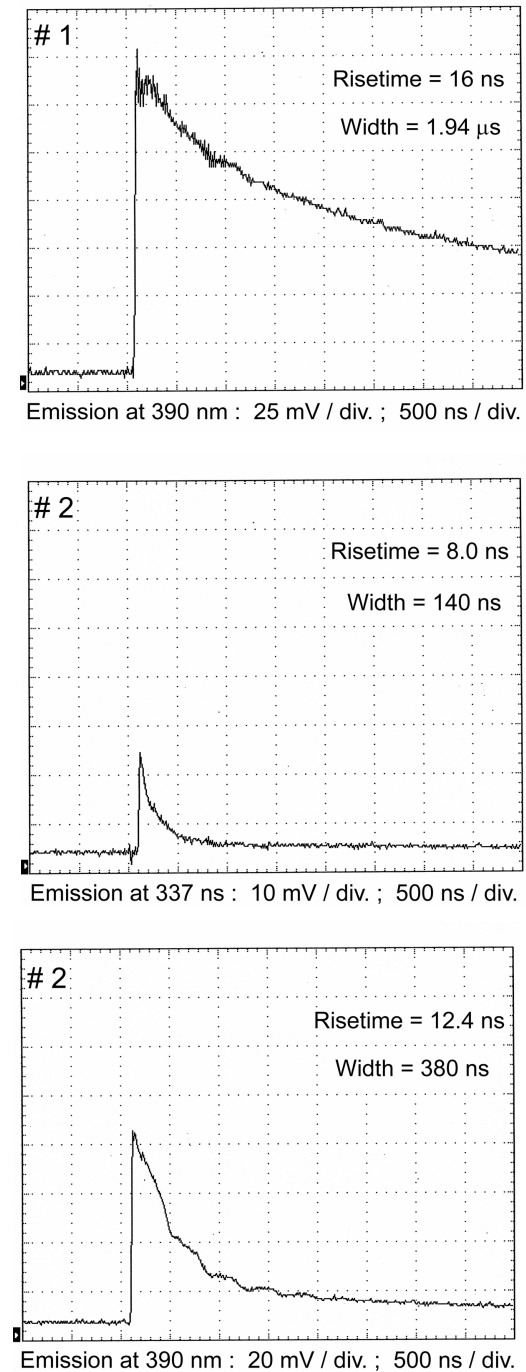
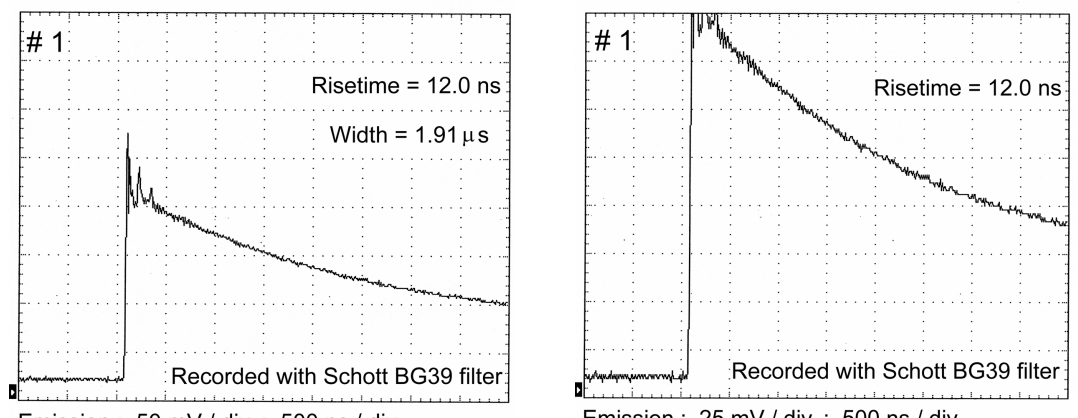

Emission : $50 \mathrm{mV} /$ div. ; $500 \mathrm{~ns} / \mathrm{div}$.
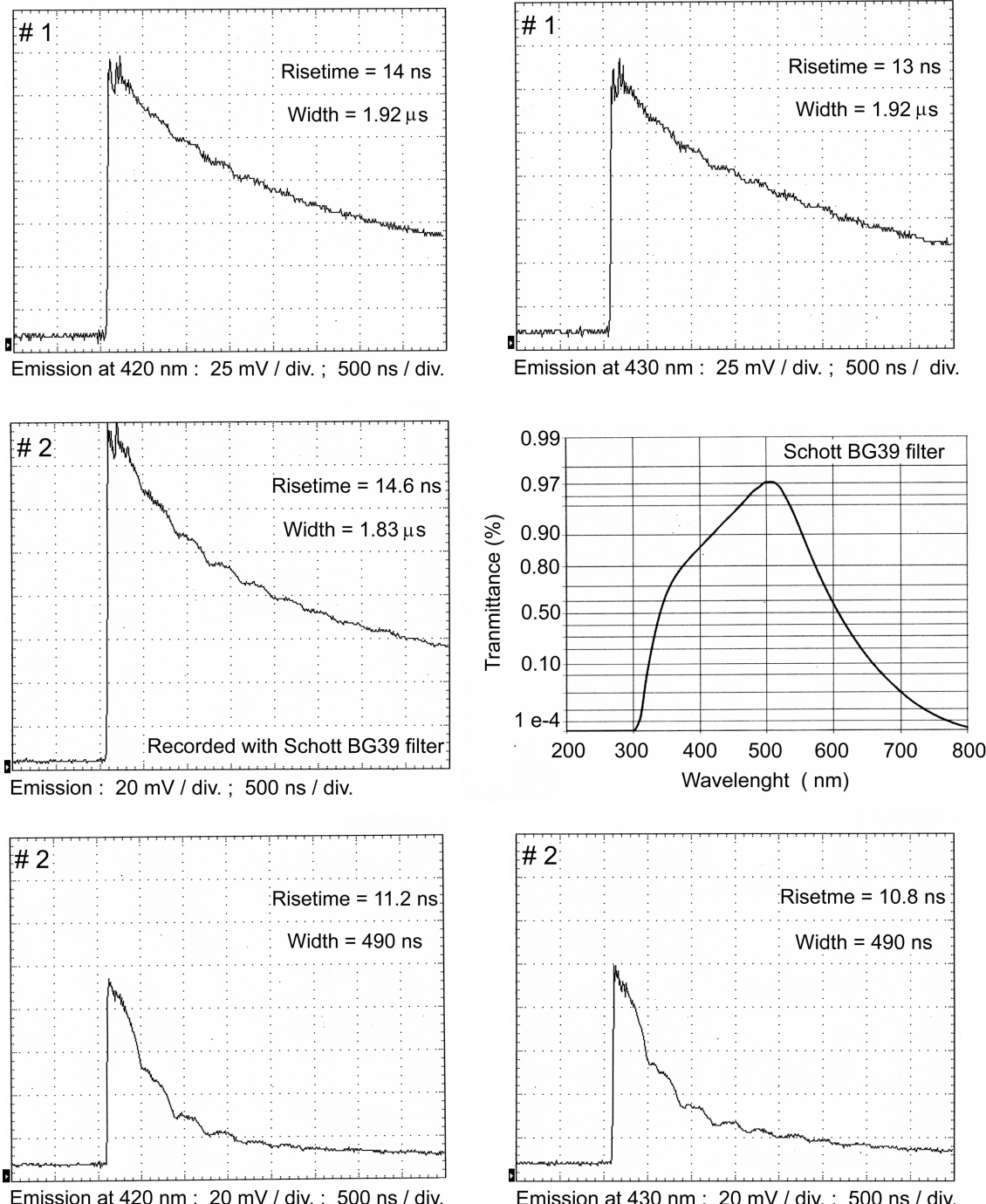

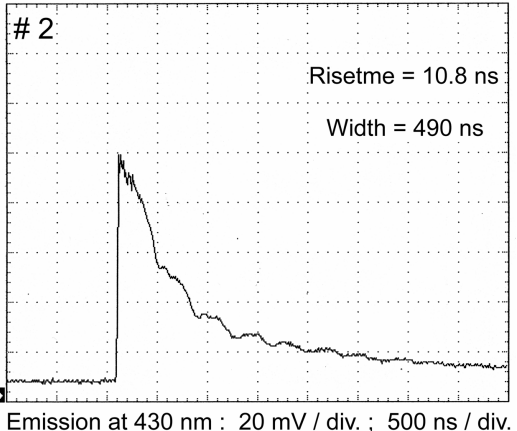

Emission at $430 \mathrm{~nm}$ : $20 \mathrm{mV} /$ div. ; $500 \mathrm{~ns} /$ div.

Fig. 3. Experimental setup to generate the emission at $337.1 \mathrm{~nm}$ of long duration. Number \#1 is for the arrangement when the photodiode is placed at the exit of the setup. Number \#2 is for the arrangement when the photodiode is placed $28 \mathrm{~cm}$ away. Three consecutive shots recorded at 337.1 nm are shown in arrangement \#1. When the radiation pulse is measured with the aid of a Schott BG 39 interference filter and observed on the 25 - $\mathrm{mV} /$ division scale, the peak amplitude of emission is $190 \mathrm{mV}$ in arrangement \#1. The average peak amplitude of emission measured with the aid of narrowband filters (= the sum of peak amplitudes of emission recorded at $337,390,420$, and $430 \mathrm{~nm}$ and divided by 4) is $145.88 \mathrm{mV}$ in arrangement \#1. The ratio between these two quantities is $190 / 145.88=1.3024$.

The question arises why many other lines have not been taken into account: the line at $394.3 \mathrm{~nm}$ belonging to the second positive system of a nitrogen molecule, as studied in [10], and the line at $471 \mathrm{~nm}$ belonging to the first negative band of $\mathrm{N}_{2}^{+}$ions, and (why not) many new vibrational bands in $\mathrm{N}_{2}$ laser emission spectra, as stated in [11].

To answer this question, the detailed analysis of the data obtained with the four narrowband filters and with the 

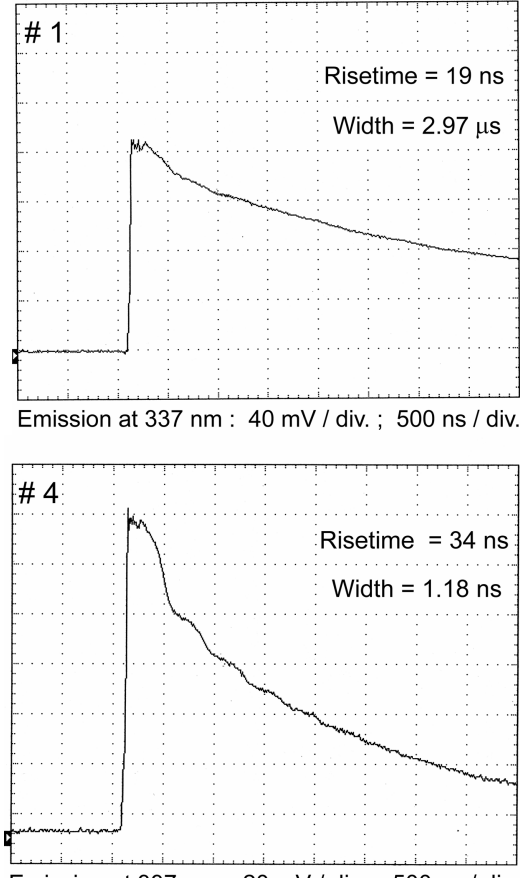

Emission at $337 \mathrm{~nm}$ : $20 \mathrm{mV} /$ div. ; $500 \mathrm{~ns} /$ div.

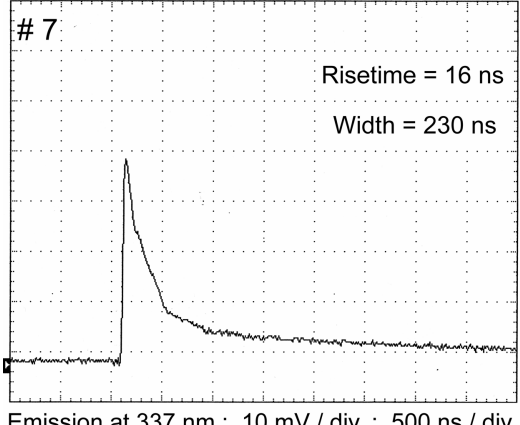

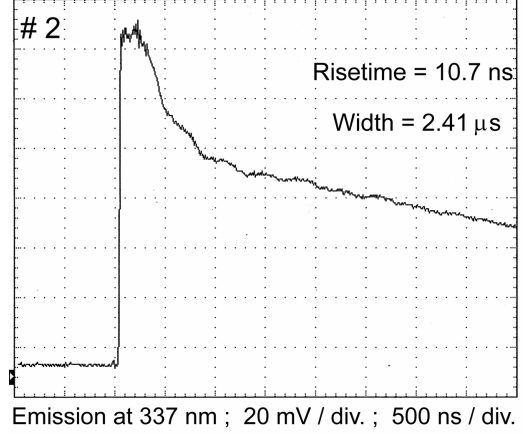

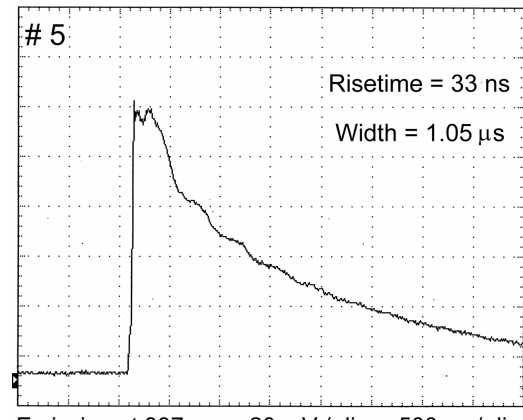

Emission at $337 \mathrm{~nm}: 20 \mathrm{mV} /$ div. ; $500 \mathrm{~ns} / \mathrm{div}$

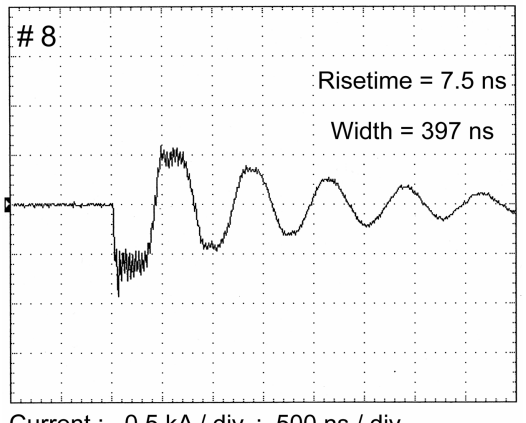

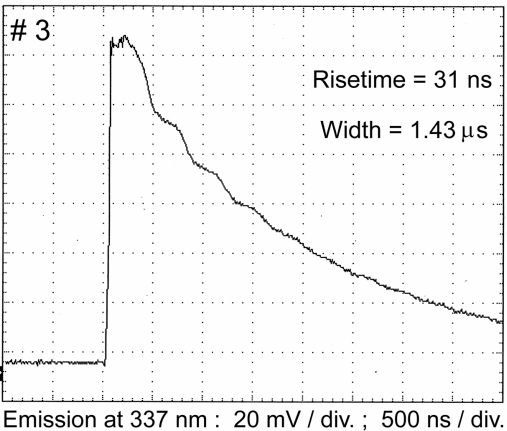

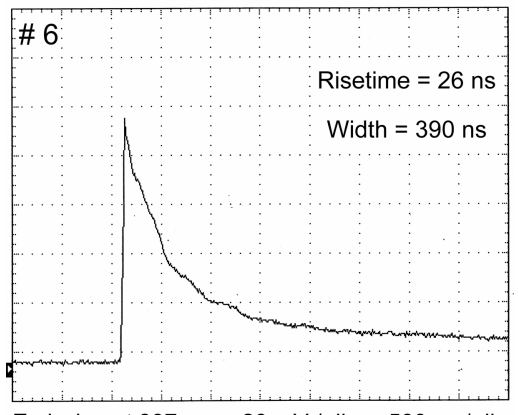

Emission at $337 \mathrm{~nm}: 20 \mathrm{mV} /$ div. ; $500 \mathrm{~ns} / \mathrm{div}$.

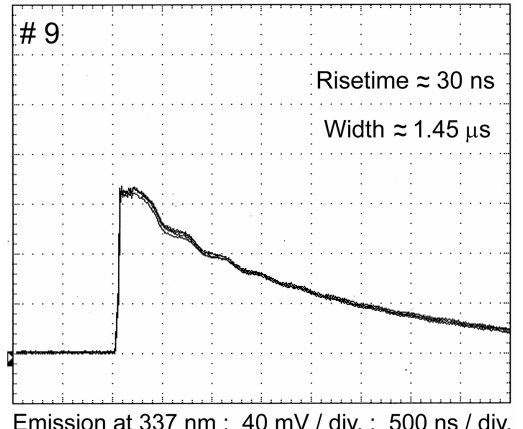

Fig. 4. Experimental setup to generate the emission at $337.1 \mathrm{~nm}$ of long duration. Seven shots of laser emission at $337.1 \mathrm{~nm}$, recorded as a function of the distance measured as the distance between the upper surface of the Plexiglas flange 3 shown in Fig. 1 and the photodiode. The distance for each frame is noted in Fig. 5. A typical current waveform is given in frame 8. Four waveforms of emission are shown in frame \#9. The emission shown in frame \#3 is also presented in frame \#9, where four shots were superimposed and the vertical scale of $20 \mathrm{mV} / \mathrm{div}$. was used in frame \#3. In frame \#9, the scale of $40 \mathrm{mV} / \mathrm{div}$ was used.

wideband filter was carried out, and the analysis will be presented later.

The data obtained are summarized in Table I. For the results given in Fig. 2, arrangement \#2, the peak amplitude of the emission measured with the aid of Schott BG39 wideband filter is $143.2 \mathrm{mV}$. The average peak amplitude of the emission (i.e., the sum of peak amplitudes of emission recorded with the aid of narrow band filters at 337, 390, 420, and $430 \mathrm{~nm}$ divided by 4) is $109.5 \mathrm{mV}$. The ratio between these two quantities is $143.2 / 109.5=1.3078$.

Similarly, for Fig. 3, arrangement \#1, the peak amplitude of emission measured with the aid of a Schott BG39 wideband filter is $190 \mathrm{mV}$. The average peak amplitude of emission (= the sum of peak amplitudes of emission recorded with the aid of narrow band filters at 337, 390, 420, and $430 \mathrm{~nm}$ divided by 4 ) is $145.88 \mathrm{mV}$. The ratio between these two quantities is $190 / 145.88=1.3024$.
It is interesting to note that when a Schott BG 39 filter is used in arrangement \#2 of Fig. 2, it was observed that the laser beam was seen illuminating the ceiling. To the naked eye, the perfect circle of blue color light of less than $10 \mathrm{~cm}$ in diameter was observed. The distance between the ceiling and the Schott BG39 filter was $75 \mathrm{~cm}$.

Also, by noting the sensitivity of the Hamamatsu R 727 photodiode versus wavelength, it can be stated that the emission at $337.1 \mathrm{~nm}$ has the larger amplitude in comparison to the amplitude recorded at 390, 420, and $430 \mathrm{~nm}$.

In arrangement \#2 given in Fig. 3, we have a sharp decrease of the emission, particularly at $337.1 \mathrm{~nm}$ when the measurement of emission is made at the distance of $28 \mathrm{~cm}$ away from the exit. To understand this observation, a detailed study was carried out. Seven waveforms of emission recorded as a function of distance formed between the laser source and the photodiode are given in Fig. 4. The distance between the laser 
TABLE I

Maximum Value of the Emission as the Function of the Filter’s WaVelength

\begin{tabular}{|l|c|c|c|c|c|}
\hline $\begin{array}{l}\text { Wavelength of the filter in [nm] used to get Fig. 2 and } \\
\text { Fig. 3 }\end{array}$ & 337.1 & 390 & 420 & 430 & BG 39 filter \\
\hline $\begin{array}{l}\text { Maximum value of the emission in [mV]. The data are } \\
\text { from Fig. 2, arrangement \# 2 }\end{array}$ & 111.2 & 107.2 & 104 & 115.2 & 143.2 \\
\hline $\begin{array}{l}\text { Maximum value of the emission in [mV]. The data are } \\
\text { from Fig. 3, arrangement \# 1 }\end{array}$ & 143 & 150 & 150 & 141.5 & 190 \\
\hline
\end{tabular}
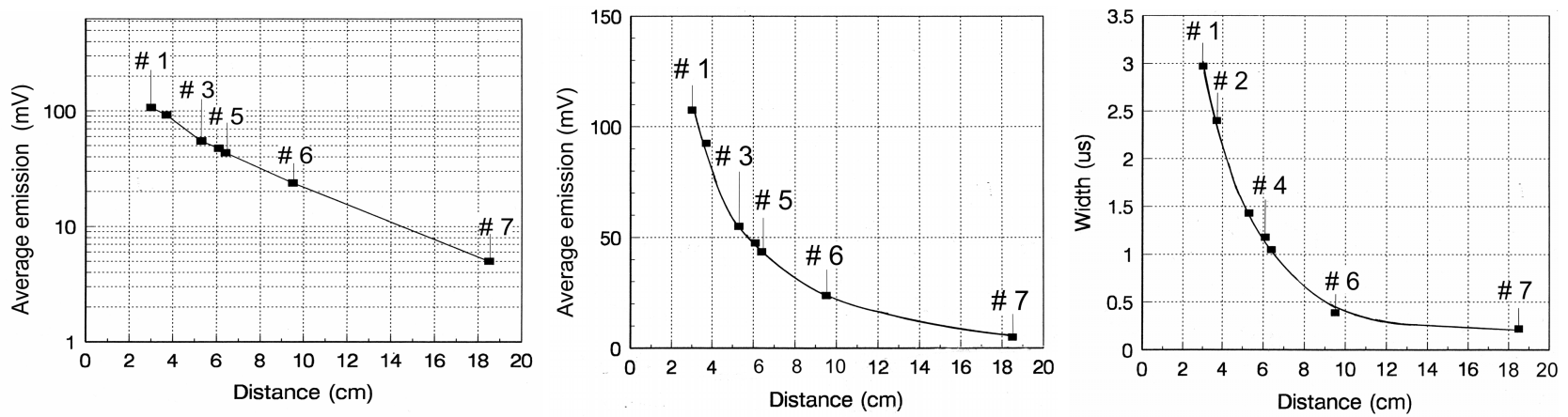

Fig. 5. Evaluation of data that are given in Fig. 4. Left: average emission versus the distance presented on the log-linear scale. Center: average emission versus the distance presented on the log-linear scale. Right: width ( = FWHM) of the pulse versus distance. The average emission value was obtained by integrating the waveform of the emission and by dividing this integral with the duration of the emission, as observed on the waveforms using a 500-ns/div. time scale.

source and the detector (photodiode) for each shot is noted on the $x$-axis given in Fig. 5. The average value of emission was evaluated by integrating the waveform of the emission and by dividing this integral with the duration of the emission, as recorded with a 500-ns/div. time scale.

The average emission presented on the log-linear scale in Fig. 5, on the left, shows that the emission decays exponentially with distance, $d$

$$
\text { Average emission }(\mathrm{mV})=A \exp .\left(-d / l_{v}\right)
$$

where $A$ is evaluated to be $144 \mathrm{mV}$ and $l_{v}$ defined as the absorption length is $5.507 \mathrm{in} \mathrm{cm}$. The decay of emission can be attributed to the plasma formation created in air by the $\mathrm{N}_{2}$-stimulated (laser) beam.

\section{THEORY}

To appreciate the data recorded with the aid of a Schott BG 39 filter, it may be useful to begin observing the interaction between two signals of same amplitude (for example, representing two electric fields) of different frequencies $f_{1}$ and $f_{2}$ that are governed by the law of coherence [12]

$$
\Delta T * \Delta F=1, \quad \text { where } \Delta F=\left\|f_{1}-f_{2}\right\| .
$$

Here, $\Delta T$ is the time interval at which the resulting signal has zero value between the two consecutive interactions. $\Delta T$ keeps repeating in time as long as the signals $f_{1}$ and $f_{2}$ are applied.

In general, when two pulses start interacting, the resulting pulse will have a very different shape in comparison to the pulses that are interacting. This fact was illustrated by the seven examples done to account for the interactions between two microwaves generated in air, and a comparison was made between the experimental data and the theory [13]. Because the energy balance must be maintained, in the ideal case, the energy contained in each pulse should be transferred to the resulting pulse.

To analyze the data given in Figs. 2 and 3, the following explanation may be necessary. The sensitivity of the photodiode is specified in $\mathrm{mA} / \mathrm{W}$, as the photodiode produces the current in response to the power it receives. This current enters $50-\Omega$ input impedance of the oscilloscope and the emission is registered in millivolts. To use expression (3) and the data given in Figs. 2 and 3, we must consider using the square root of emission recorded. It is useful to divide the frequencies of all wavelengths by a factor of $10^{6}$, so that the Pspice program can be used. Because the power is measured using the four filters at $337.1,390,420$, and $430 \mathrm{~nm}$, we must have four independent circuits that are capable of generating longduration pulses (from $20 \mathrm{~ns}$ to $1 \mu \mathrm{s}$ ) of constant amplitude. Frames \#1 and \#2 in Fig. 6 correspond to the wavelength of $337.1 \mathrm{~nm}$ for the data presented in Fig. 2. Because we are not certain about the possible phase shift among the frequencies during the interaction, it is preferable to sum up the power of each signal while the interaction takes place. The square root of the peak amplitude of the emission recorded at a particular wavelength is inserted in each circuit. Afterward, the square root of emission is multiplied by itself and also by the transmittance of the Schott BG 39 interference filter for this particular wavelength. This procedure is repeated for all the four circuits, and the result was summed up using the averaging function of the Pspice program. The result obtained is now divided by the average peak amplitude of emission (i.e., the sum of peak amplitudes of emission recorded at 337, 


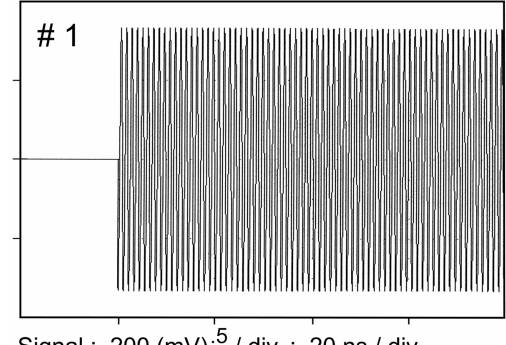

Signal : $200(\mathrm{mV})^{.5} /$ div. ; $20 \mathrm{~ns} / \mathrm{div}$.

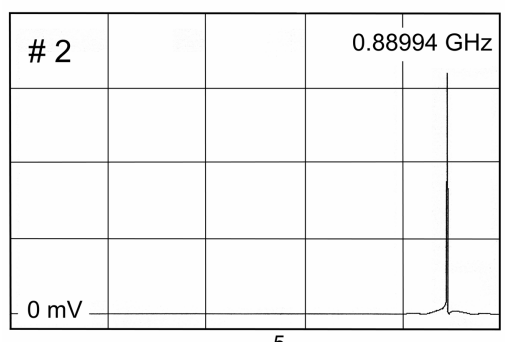

FFT of signal : $(100 \mathrm{mV})^{.5} /$ div. ; $0.2 \mathrm{GHz} /$ div.

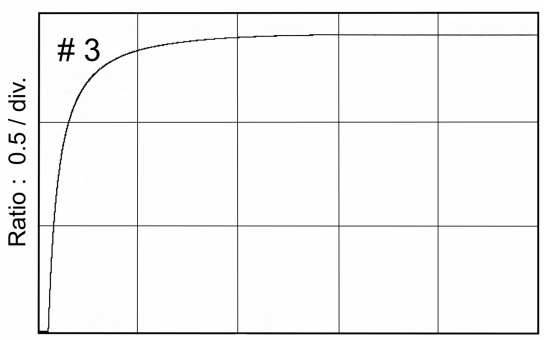

Time : $200 \mathrm{~ns} / \mathrm{div}$.

Fig. 6. Evaluation of the ratio between the peak amplitude of the emission that should be measured with the aid of a Schott BG 39 wideband filter and the average peak amplitude of the emission (i.e., the sum of peak amplitudes of the emission recorded at 337, 390, 420, and $430 \mathrm{~nm}$ and divided by 4 ) obtained in the experiments. The Pspice program shows that this ratio reaches the maximum value of 1.4105 at the time close to $1 \mu \mathrm{s}$. This value differs from the ratio given in Fig. 2 by $7.52 \%$. Frames \#1 and \#2 give the waveform of the signal and its FFT. They correspond to the square root of the peak amplitude of the emission recorded at $337.1 \mathrm{~nm}$ shown in Fig. 2. To use the Pspice program, the frequencies of all the wavelengths considered were divided by a factor of $10^{6}$.

390,420 , and $430 \mathrm{~nm}$ and divided by 4). The values of the peaks shown in Fig. 2 for arrangement \#2 were used in the analysis. With the aid of the Pspice program, shown in Fig. 6, frame \#3 was obtained and it shows that the ratio reaches the maximum value of 1.4095 at the time close to $1 \mu \mathrm{s}$. In Fig. 2, arrangement \#2, the ratio of Fig. 3 of arrangement \#1 was used in the Pspice program, and an almost identical result of the calculated ratio was obtained. In Fig. 3, the experiments show that the experimental value of the ratio is 1.3024.

If the amplitude generated by the independent circuits is made to fall by a few percent, then the curve describing the ratio displays the maximum before $1 \mu \mathrm{s}$ and the maximum value of the ratio becomes closer to the measured value of the ratio of 1.3078. The short-duration spikes occurring after the onset of emission and being present on top of all waveforms, as recorded in Fig. 3, for arrangement \#1, and particularly the data recorded with the Schott BG 39 interference filter using the $50 \mathrm{mV} / \mathrm{div}$. scale are not included in the calculation of the ratio. The peak amplitude of emission was averaged by taking the midpoint value of these spikes.

If the calculated ratio would have been of substantially smaller value, in comparison to the one recorded in the experiments, it would mean that some additional radiation lines should be considered. Hence, it is appropriate to state that four narrow band filters at 337, 390, 420, and $430 \mathrm{~nm}$ were sufficient to account for the emission output of the device during the average peak of the emission.

\section{DISCUSSION}

Figs. 4 and 5 provide the answer why the emission at $337.1 \mathrm{~nm}$ has such small value at some distance from the exit. It is believed that the high-power level of the 337.1-nm line close to the exit has photoionized air molecules to a sufficient degree to create the plasma. The radiation beam is not focused and the plasma is not bright enough to be named the laser spark. The laser spark is formed when the beam is focused by the lens.

When the laser beam is of femtosecond duration, many studies were done to determine the population inversion in the femtosecond plasma and the lasing action [14], [15].
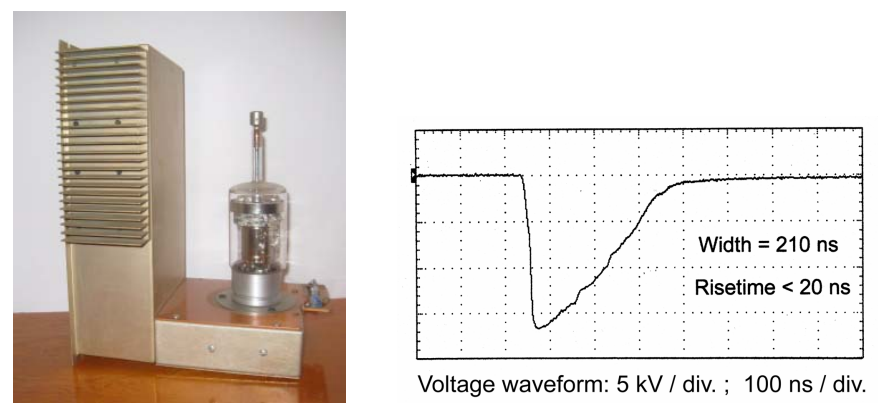

Voltage waveform: $5 \mathrm{kV} / \mathrm{div}$; $100 \mathrm{~ns} / \mathrm{div}$.

Fig. 7. Basic part of the CX unit showing the thyratron on the right and the associated circuit placed in the enclosure (left). The energy storage network (i.e., capacitor, and so on) was not yet attached. Right: voltage waveform produced by the $\mathrm{CX}$ unit.

The visible blue radiation recorded by 390-, 420-, and 430-nm filters shown in Fig. 3, for arrangement \#2, may be in part due to the radiation of the newly formed plasma. There is also the possibility that the radiation coming from the $\mathrm{N}_{2}$ laser just penetrates the plasma. The high amplitude recorded by the Schott BG 39 filter suggests that the plasma is producing its own lines, because the emission recorded by the narrow band filters at 390,420 , and $430 \mathrm{~nm}$ are of moderately high amplitude.

Without using the filter at the exit of the $\mathrm{N}_{2}$ laser, still photographs of visible blue radiation were obtained. They show that the radiation is emitted in the form of a cone having the wide angle of $>30^{\circ}$ in respect of the axis with the largest amplitude (brightness) still at the axis.

During these experiments, it became evident of the need to visualize the photoexcitation and photoionization processes without the use of sophisticated diagnostics. To achieve this goal, the thyratron trigger unit producing the voltage pulse given in Fig. 7 was used to induce the discharges in the Plexiglas cylinder of $140 \mathrm{~mm}$ diameter at low pressures $(\sim 1$ torr) in air (Figs. 8 and 9 ).

In the cylinder, the two copper electrodes were bent to form a gap of $3.2 \mathrm{~cm} .12 \mathrm{~cm}$ away above this gap, the copper electrode of $3.5 \mathrm{~mm}$ in diameter was placed (see Fig. 8). The pulse from the CX trigger unit was split into two parts. The gap of $3.2 \mathrm{~cm}$ receives the voltage pulse via a $300-\mathrm{pF}$ 


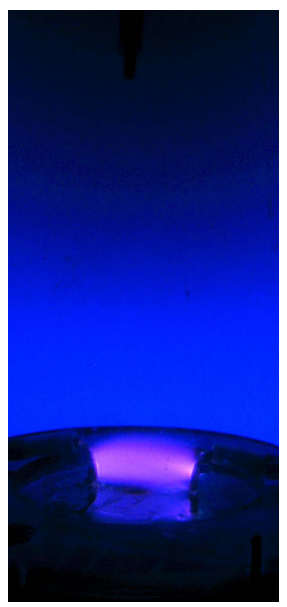

\# 1

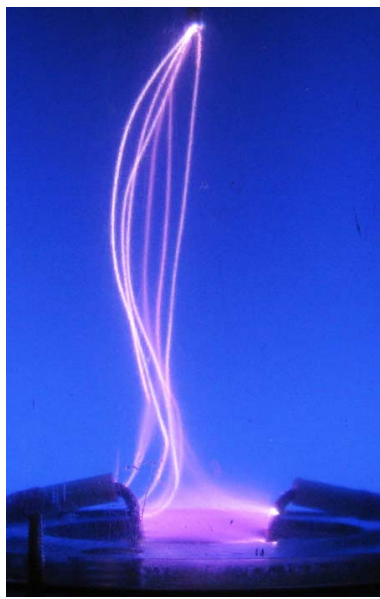

\# 4

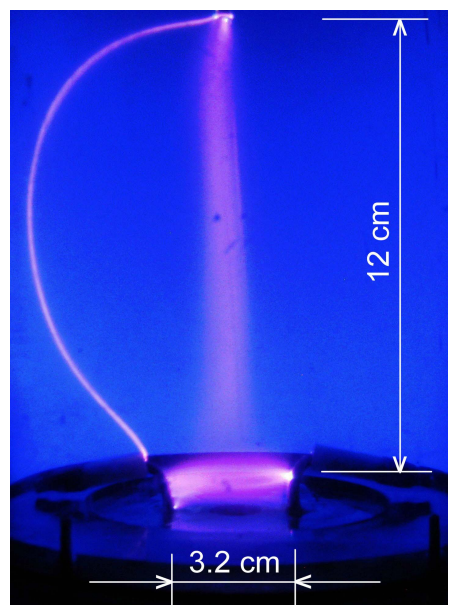

\#2

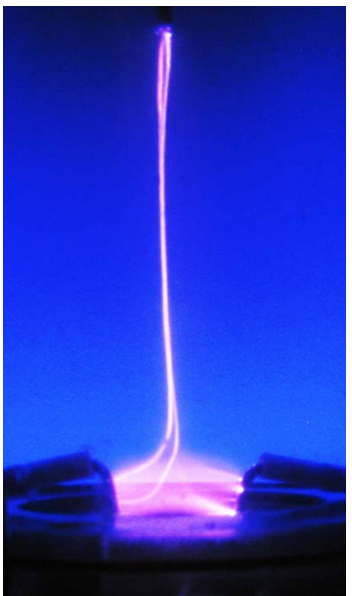

\# 5

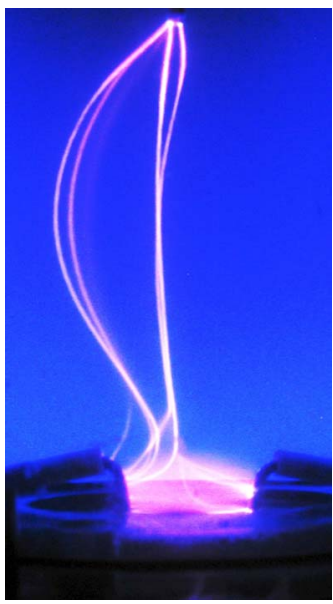

\# 3

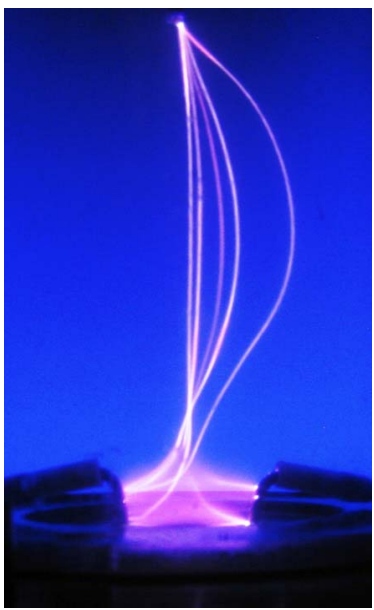

\# 6

Fig. 8. Six still photographs of the discharge in air at about 1 torr obtained by using a (pocket size) Cannon camera: SD 870 IS. The two copper electrodes shown at the bottom were bent to form the gap of $3.2 \mathrm{~cm}$. The electrode on the left was at the ground potential. The gap of $3.2 \mathrm{~cm}$ receives the voltage pulse via a 300-pF capacitor. $12 \mathrm{~cm}$ above this gap, the copper rod was placed. It was connected directly to the CX unit operating up to 10 pulses/s.

capacitor and the copper electrode is connected directly to the unit. By setting the unit to operate at $<10$ pulses per second, the cylinder was gradually evacuated until the spark breakdown would occur between the two curved electrodes. First, the spark channels would glide along the surface and as the pressure in the chamber would decrease close to 1 torr, its path becomes concave. The visible blue light now illuminates the lower part of the chamber to above 5-6 cm above the bottom plate. To be sure that this emission was not partially due to the voltage, the connection to the rod was severed and pictures were recorded.

Afterward, the copper rod was connected to the CX trigger unit. By varying the pressure by a small fraction of 1 torr, the pictures of the discharges were recorded, as shown in Fig. 8, \#2-6. First, the diffused glow from the rod toward the discharge between the curved electrodes was making the visible blue light to be well present in the whole chamber.

In Fig. 8, we also have the filamentary discharge (spark channel-like discharge), as it traverses first toward the wall of the Plexiglas cylinder, then makes an arc before reaching the electrode on the left which is on the ground potential. This behavior is also evident in pictures \#2-\#4. Picture \#5 is rather an exception to the rule to have nearly straight spark channellike discharges in the vertical direction advancing from the rod to the discharges formed between two curved electrodes.

The trajectory of the filamentary discharges is governed by the local electric field at the tip of the developing channel and by the conductivity ahead of the tip in the glow discharge. From the pictures in Fig. 8, it can be suggested that the conductivity in the radial direction can be of large value in comparison to that in the vertical direction in some shots, and higher conductivity in the radial direction makes the channel to bend toward the wall. The enhancement of conductivity comes from the UV radiation being generated by the glow discharge, as it induces the electron emission from the wall of the Plexiglas cylinder and makes the surface of the insulator positively charged. The overall effect is to force the filamentary channel to bend on the right, as shown in picture \#6, or on left, as indicated in pictures \#2-4 of Fig. 8. 

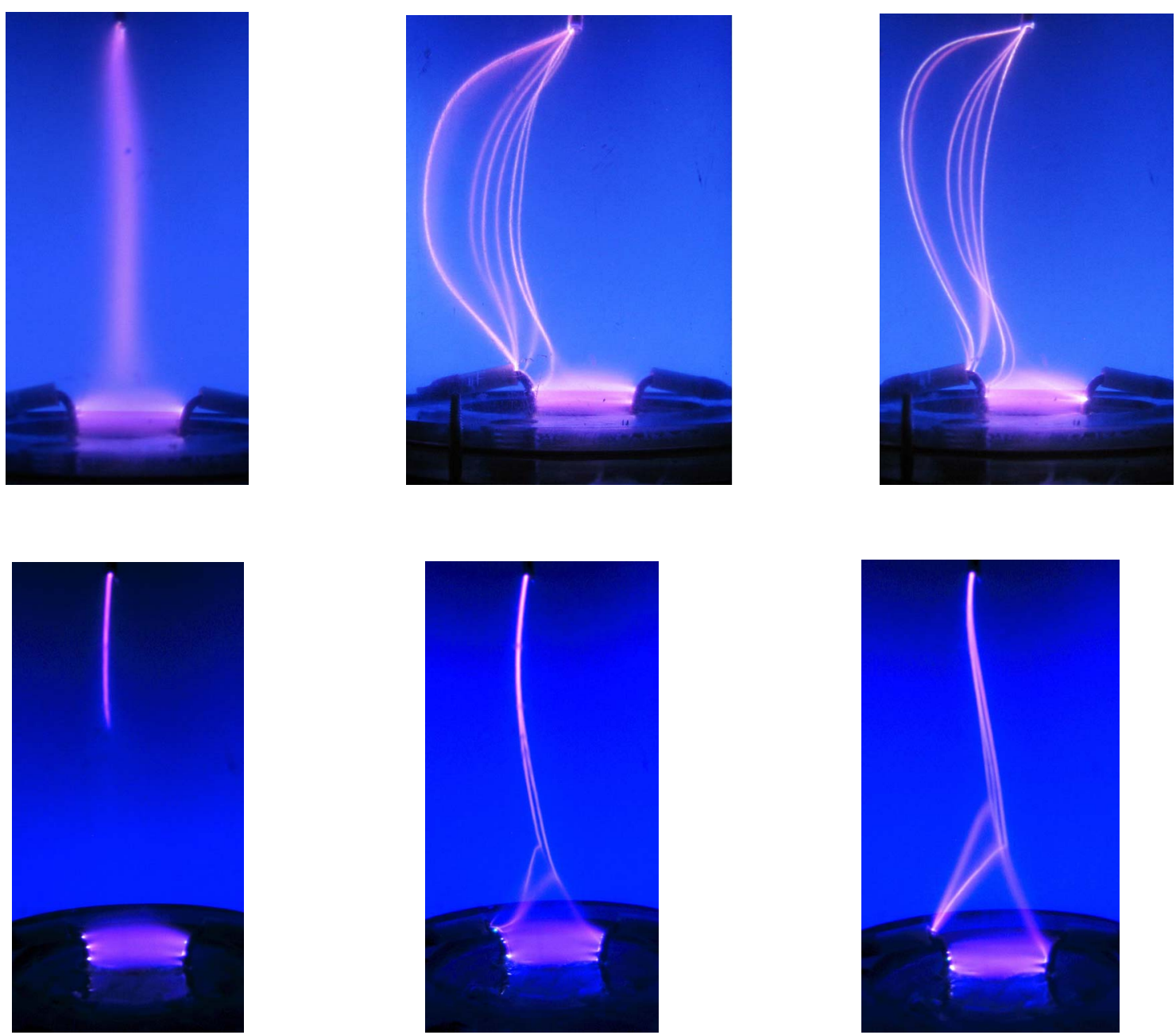

Fig. 9. Three photographs in the top row were obtained under the same conditions as in Fig. 8. The three photographs at the bottom row were recorded under the condition when the connections were reversed, i.e., the "hot" lead from the CX unit was attached to the curved electrode given on the left and the "ground" lead was applied to the rod. The gap of $3.2 \mathrm{~cm}$ receives the voltage pulse from the CX unit via $300 \mathrm{pF}$.

To examine this hypothesis, the connection from the CX unit to the chamber was reversed. The rod was made to be at ground potential and the curved electrode given on the left was subjected to the pulse of negative polarity. The gap of $3.2 \mathrm{~cm}$ still receives the voltage pulse via the $300-\mathrm{pF}$ capacitor. The data obtained are given in Fig. 9 (bottom row).

The brightness of the discharge in the pictures given in Fig. 9 shown at the bottom row is smaller than that given in the top row; hence with the reverse connections, the filamentary channel follows more or less the path of the electrostatic field line.

The question whether to apply the reverse polarity or not comes from the earlier experiences gained during the development of the UV-preionized $\mathrm{CO}_{2}$ lasers. It was learned that when the UV sources are situated behind a mesh anode and the cathode receives the voltage pulse of negative polarity, efficient (bright) glow discharge is generated. Here, the goal is to get bright glow discharges formed between the two Rogowski profiled electrodes to act as the laser gain medium. Fig. 10(a) shows the picture of the section of the UV-preionized $\mathrm{CO}_{2}$ laser that has been developed in the laboratory. The development of a 300-J multigigawatt $\mathrm{CO}_{2}$ laser was described in [17].
The processes shown in Fig. 8, picture \#1, may take place at high altitudes at $>60 \mathrm{~km}$ above the Earth. The sun emits a significant amount of UV radiation (about 10\% of its total power) and the UV and shorter wavelengths are capable of dislodging an electron from a neutral gas molecule/atom during the collision via photoionization. Also, the photoexcitation of a neutral molecule takes place. In addition to UV radiation, the electrons, protons, and alpha particles blown toward the earth in the solar wind dictate the earth environment. These radiations and particles are absorbed mainly by nitrogen, and they can pump the $\mathrm{N}_{2}$ molecules to the upper, excited, $\mathrm{C}^{3} \Pi_{u}$ level and at longer wavelengths by simple diatomic oxygen. Most of the remaining UV in the midrange of energy is blocked by the ozone layer.

Without the external signal to trigger the onset of sprites, the excited state of $\mathrm{N}_{2}$ molecules will decay via the spontaneous emission, hence creating the temperature profile across the mesosphere and giving the peak in the temperature at $48 \mathrm{~km}$ above the earth. At high altitudes, the spontaneous emissions should be of the same color as those shown in Fig. 8, picture \#1, and should be coming from the first negative 


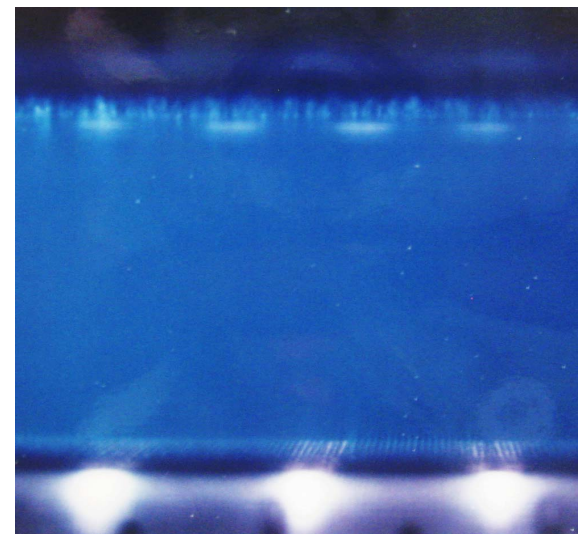

(a)

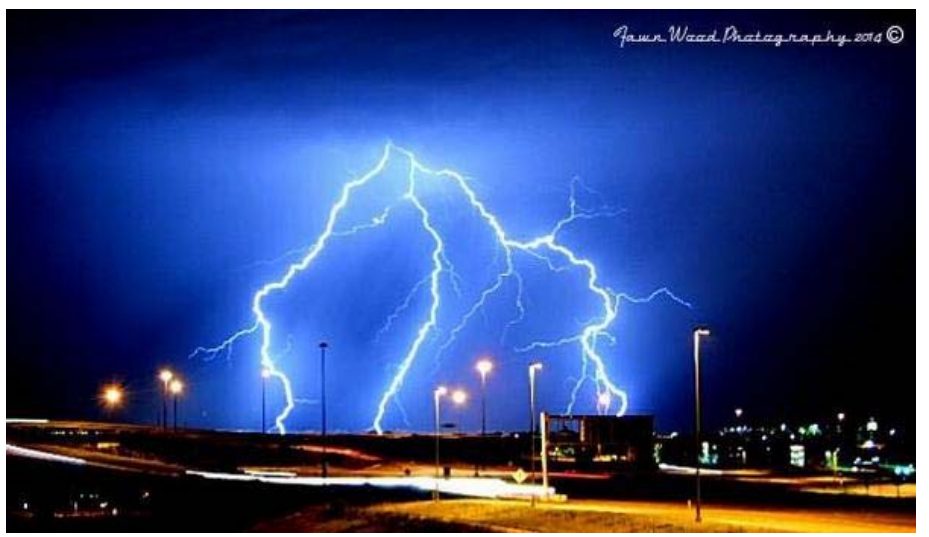

(b)

Fig. 10. (a) Still photograph of the $\mathrm{CO}_{2}$ laser discharge in the mixture: $30 \%$ (240 torr) of $\mathrm{CO}_{2}, 20 \%$ (160 torr) of $\mathrm{N}_{2}$, and $50 \%$ (400 torr) of He. The separation between the two parallel Rogowski-shaped electrodes was $10 \mathrm{~cm}$. Car spark plugs were used as UV sources and they were placed $5 \mathrm{~cm}$ below the mesh anode, which was on the ground potential. The $\mathrm{CO}_{2}$ laser has generated the laser-created plasma presented in [16]. (b) Photograph of cloud to ground lightning recorded by Fawn Wood Photography, USA, under the conditions of low humidity in air. Every short length of the lightning bolt in the processes known as the return strokes creates the nitrogen laser emission, and the visible blue laser line illuminates a large area.

band of $\mathrm{N}_{2}^{+}$ions due to the $\mathrm{B}^{2} \sum_{u}^{+} \rightarrow \mathrm{X}^{2} \sum_{g}^{+}$transition at 391.4 and $427.8 \mathrm{~nm}$.

The photograph of cloud to ground lightning is shown in Fig. 10(b). It could be suggested that every short length of the lightning bolt in the processes known as the return strokes may create the nitrogen laser emission, and the visible blue laser line will illuminate a large area around the return strokes [see Fig. 10(b)].

Fig. 5 points out that the absorption length has a finite dimension and Fig. 3, arrangement \#2, shows the partial escape of the laser beam at $337.1 \mathrm{~nm}$ from the rear of the plasma due to insufficient absorption.

These facts have the bearing on the generation of $\mathrm{N}_{2}$ laser emission and the creation of sprites and elves [6]. The size of the sprite was estimated initially using the Chapman-Jouguet condition for the detonations [18], [19]

$$
v^{2}=\left[2\left(\gamma^{2}-1\right)\right] E_{M}
$$

where $\gamma$ is the adiabatic index, $v$ is the detonation velocity, and $E_{M}$ is the energy per unit mass. Here, the absorption length is considered to have a small value. After cessation of the laser beam, the detonation wave will decay into the shock wave and then into the sound wave. The size of the sprite was specified when the velocity of the detonation wave became equal to the sound speed.

Many studies were carried out to examine the radiative detonation model for the development of a laser-induced spark. In [20], the laser-supported detonations with large absorption lengths were examined. Using the Chapman-Jouguet condition again, the analysis yields the following interpretation. With large absorption lengths, the velocity of laser spark elongation is of larger value, as compared to the case when the absorption length is small. This implies that ahead of the detonation front, we have the breakdown wave, i.e., a pure phase velocity without mass motion (hence, no Doppler effect). The net result of having the absorption length means that the energy content of the sprites observed has the smaller value of the energy, in comparison to the energy needed when the absorption length is short as it was done in [6].

\section{CONCLUSION}

The intense $\mathrm{N}_{2}$ laser pulses at $337.1 \mathrm{~nm}$ of long duration $(>2 \mu \mathrm{s})$ are subject to strong attenuation as the beam traverses in air.

The energy of the high-power beam at $337.1 \mathrm{~nm}$ induces intense multiphotoionization of air molecules and the excitation of molecules and ions to high-lying excited states. This process leads to the creation of the plasma, at a short distance away from the exit. Fig. 2 indicates that the $\mathrm{N}_{2}$ laser beam at $337.1 \mathrm{~nm}$ of short duration $(\sim 10 \mathrm{~ns})$ is also subject to a smaller degree of attenuation.

By using the wideband Schott BG 39 filter and the Pspice program, it was demonstrated that the narrow band filters at $337.1,390,420$, and $430 \mathrm{~nm}$ can account for the overall emission in the setup tuned to generate the short pulses at $337.1 \mathrm{~nm}$ (see Fig. 2) as well as for the setup in Fig. 3 organized to generate long-duration pulses at $337.1 \mathrm{~nm}$ only at the distance close to the exit.

In the experiments with the thyratron trigger unit, UV photoionization is demonstrated. A visible blue emission line from the molecular nitrogen positive $\left(1^{+}\right)$ion is responsible for the blue color observed in Figs. 8 and 9 and may offer an additional explanation why the sky is blue on a clear day.

The purpose of conducting this research and development is also to get the background information that is required to help design the explosively driven magnetocumulative generator (MCG), capable of producing the train of $\mathrm{N}_{2}$ laser pulses, necessary for high-altitude studies of sprites in order to help us understand better the origin of the Tunguska event [6]. By introducing the concave mirror, it was demonstrated that the current experimental setup is capable of producing the laser spark, i.e., the laser-induced electrical breakdown in atmospheric air. 


\section{REFERENCES}

[1] U. Rebhan, J. Hildebrandt, and G. Skopp, "A high power $\mathrm{N}_{2}$-laser of long pulse duration," Appl. Phys., vol. 23, no. 4, pp. 341-344, Dec. 1980.

[2] D. Basting, F. P. Schäfer, and B. Steyer, "A simple, high power nitrogen laser," Opto-Electron., vol. 4, no. 1, pp. 43-49, Feb. 1972.

[3] A. Martinez and V. Aboites, "High-efficiency low-pressure Blumlein nitrogen laser," IEEE J. Quantum Electron., vol. 29, no. 8, pp. 2364-2370, Aug. 1993.

[4] V. F. Tarasenko, "Efficiency of a nitrogen UV laser pumped by a selfsustained discharge," Quantum Electron., vol. 31, no. 6, pp. 489-494, Jun. 2001.

[5] M. M. Kekez, "Laser and microwave generations in nitrogen," IEEE Trans. Plasma Sci., vol. 46, no. 3, pp. 545-555, Mar. 2018, doi: 10.1109/ tps.2018.2796304.

[6] M. M. Kekez, "Generation of nitrogen emission line at $337 \mathrm{~nm}$ in the laboratory and at high altitudes," IEEE Trans. Plasma Sci., vol. 47, no. 6, pp. 2801-2811, Jun. 2019, doi: 10.1109/tps.2019.2914564.

[7] N. S. Kopeika and A. P. Kushelevsky, "The role of excited atoms in UV photo-preionization TEA lasers," IEEE J. Quantum Electron., vol. QE-13, no. 12, pp. 968-972, Dec. 1977.

[8] H. Seguin, J. Tulip, and D. Mcken, "Ultraviolet photoionization in TEA lasers," IEEE J. Quantum Electron., vol. QE-10, no. 3, pp. 311-319, Mar. 1974.

[9] O. D. Judd and J. Y. Wada, "Investigations of a UV preionized electrical discharge and $\mathrm{CO}_{2}$ laser," IEEE J. Quantum Electron., vol. QE-10, no. 1, pp. 12-20, Jan. 1974.

[10] M. I. Lomaev, D. V. Rybka, D. A. Sorokin, V. F. Tarasenko, and K. Y. Krivonogova, "Radiative characteristics of nitrogen upon excitation by volume discharge initiated by runaway electron beam," Opt. Spectrosc., vol. 107, no. 1, pp. 33-40, Jul. 2009, doi: 10.1134/ $0030400 x 09070054$

[11] N. Subhash, S. C. Kartha, and K. Sathianandan, "New vibrational bands in nitrogen laser emission spectra," Appl. Opt., vol. 22, no. 22, p. 3612 , Nov. 1983, doi: 10.1364/ao.22.003612.

[12] P. A. Tipler, Physics, 2nd ed. New York, NY, USA: Worth Publishers, Nov. 1983, pp. 454-456.

[13] M. M. Kekez, "Interactions between two microwaves generated in air and $\mathrm{SF}_{6}$ /argon mixture," IEEE Trans. Plasma Sci., vol. 42, no. 6 , pp. 1573-1585, Jun. 2014.

[14] Y. Liu, S. Mitryukovskiy, P. Ding, A. Houard, and A. Mysyrowicz, "Lasing from plasma filaments in air," in CLEO, OSA Tech. Dig., 2015, Paper SM2N.2, doi: 10.1364/CLEO_SI.2015.SM2N.2.

[15] Y. Wei et al., "Spectroscopic analysis of high electric field enhanced ionization in laser filaments in air for corona guiding," High Power Laser Sci. Eng., vol. 4, no. 1, 2016, Art. no. 010000e8, doi: 10.1017/hpl.2016.7.

[16] M. M. Kekez and A. H. Makomaski, "Beam reentry into a laser created plasma," J. Phys. D, Appl. Phys., vol. 12, no. 5, pp. 729-738, May 1979.

[17] M. Richardson, A. J. Alcock, K. Leopold, and P. Burtyn, "A 300-J multigigawatt $\mathrm{CO}_{2}$ laser," IEEE J. Quantum Electron., vol. QE-9, no. 2, pp. 236-242, Feb. 1973.

[18] S. A. Ramsden and P. Savic, "A radiative detonation model for the development of a laser-induced spark in air," Nature, vol. 203, no. 4951, pp. 1217-1219, Sep. 1964.
[19] P. Yu Raizer, "Heating of a gas by a powerful light pulse," Sov. Phys. JETP, vol. 21, no. 5, pp. 1009-1017, Nov. 1965.

[20] M. M. Kekez and P. Savic, "Laser-supported detonations with large absorption lengths," J. Phys. D, Appl. Phys., vol. 13, no. 9, pp. L167-L170, Sep. 1980.

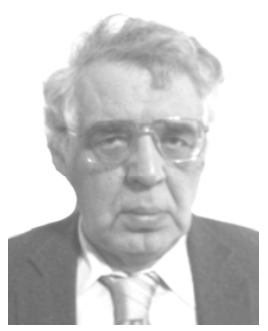

Mladen M. Kekez was born in 1941. He received the Dipl.-Ing. degree from the University of Belgrade, Belgrade, Serbia, in 1965, and the $\mathrm{Ph.D}$ degree from the University of Liverpool, Liverpool, U.K., in 1968.

From 1971 to 1972 and from 1974 to 2002, he was with the National Research Council of Canada, Ottawa, ON, Canada, doing research and development on gas discharges, pulsed power technology, plasma fusion-related experiments, biophysics, and fluid dynamics. From 1972 to 1974, he was with the University of Alberta, Edmonton, $\mathrm{AB}$, Canada, doing research and development on the $\mathrm{CO}_{2}$ lasers. In 2002, he formed the company High-Energy Frequency Tesla Inc., Ottawa, to continue with research and development. One of his research and developments was in the high-power microwave (HPM)/laser radiation in air, other gases, and gas mixture.

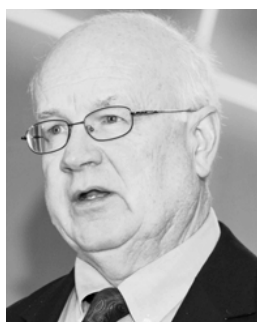

David M. Villeneuve received the Ph.D. degree in physics from the University of Waterloo, Waterloo, ON, Canada, in 1980.

After he held a post-doctoral position at the University of Rochester, Rochester, NY, USA, he joined the National Research Council (NRC), Ottawa, ON, Canada. He is currently the Head of the Attosecond Science Group, NRC, and the NRC Head of the Joint Attosecond Science Laboratory (JASLab), and the Scientific Co-Lead of the Joint Centre for Extreme Photonics, both joint undertakings between NRC and the University of Ottawa, Ottawa. He is currently an Adjunct Professor of physics with the Department of Physics, Faculty of Science, University of Ottawa. He has over 200 publications in refereed journals. His interests include the generation of attosecond-duration pulses, soft X-ray coherent imaging, high-harmonic spectroscopy, and the study of atoms and molecules in intense laser fields.

Dr. Villeneuve is a fellow of the Royal Society of Canada, the American Physical Society, and the Optical Society of America. He received the IEEE Quantum Electronics Award in 2016. 